\title{
Prognostic and staging value of tumor deposits in patients with rectal cancer after neoadjuvant chemoradiotherapy
}

\author{
Tianlei Xu ${ }^{1 \#}$, Zhuo Yu ${ }^{2 \#}$, Qian Zhang ${ }^{1}$, Botao Liu ${ }^{1}$, Yuanxin Li $^{1}$, Feng Wang ${ }^{1}$ \\ ${ }^{1}$ Department of Gastrointestinal Surgery, Beijing Tsinghua Changgung Hospital, School of Clinical Medicine, Tsinghua University, Beijing, China; \\ ${ }^{2}$ Department of Medical Oncology, Beijing Tsinghua Changgung Hospital, School of Clinical Medicine, Tsinghua University, Beijing, China \\ Contributions: (I) Conception and design: T Xu, Z Yu; (II) Administrative support: F Wang, Q Zhang; (III) Provision of study materials or patients: F \\ Wang; (IV) Collection and assembly of data: Y Li, B Liu; (V) Data analysis and interpretation: T Xu, F Wang; (VI) Manuscript writing: All authors; \\ (VII) Final approval of manuscript: All authors. \\ "These authors contributed equally to this work. \\ Correspondence to: Feng Wang, Yuanxin Li. Department of Gastrointestinal Surgery, Beijing Tsinghua Changgung Hospital, School of Clinical \\ Medicine, Tsinghua University, Beijing, China. Email: wfa02037@btch.edu.cn; surgeon_liyx@163.com.
}

Background: The presence of tumor deposits (TDs) is only considered in the absence of lymph node metastases (LNMs) in the current TNM staging system. However, the prognostic value of TDs when concomitant with LNM for rectal cancer after neoadjuvant chemoradiotherapy (NCRT) remains unclear. This study aimed to evaluate the prognostic value of TDs and when concomitant with LNMs in rectal cancer after NCRT.

Methods: Patients with rectal cancer who had received NCRT between 2010 and 2016 were obtained from the Surveillance, Epidemiology and End Results (SEER) 18 (year range, 1975-2016) database. Data were extracted on the following: age, sex, race, TNM stage, total LNs harvested, positive LNs, histologic type, perineural invasion, grade, carcinoma embryonic antigen status, TD number, and cancer-specific survival (CSS) rates. The primary objective was to determine the prognostic impact of TDs on CSS. The effect of the addition of TD to the LNM count for a novel N stage was also evaluated. Univariate and multivariate analyses were performed using the Kaplan-Meier method and Cox models.

Results: Of 9,620 patients, 865 (9.0\%) had TDs. TD-positive patients showed a worse prognosis than TD-negative patients ( $\mathrm{HR}=2.39,95 \% \mathrm{CI}: 2.04-2.80, \mathrm{P}<0.001$ ), and multivariate analysis showed that the presence of TDs was an independent poor prognostic factor (HR =1.41, 95\% CI: 1.19-1.67, $\mathrm{P}<0.001)$. Regarding the LN status, TDs were associated with a higher risk of cancer-specific death in the LNMgroup (HR =2.43, 95\% CI: 1.86-3.18, $\mathrm{P}<0.001)$, M1 group (HR =1.51, 95\% CI: $1.08-2.10, \mathrm{P}<0.001)$, and ypN1 group $(\mathrm{HR}=2.08,95 \% \mathrm{CI}: 1.61-2.70, \mathrm{P}<0.001)$, but not in the ypN2 group $(\mathrm{HR}=0.97,95 \% \mathrm{CI}$ : 0.69-1.36, $\mathrm{P}=0.84)$. Patients with concomitant TDs and LNM showed significantly worse survival than those with TDs or LNM alone (5-year CSS: 48.2\%, 72.2\%, and 67.8\%, respectively). The 5-year CSS rates were $86.2 \%, 77.4 \%, 65.1 \%, 53.8 \%$, and $46.5 \%$ for the novel N0, N1a, N1b, N2a, and N2b groups, respectively $(\mathrm{P}<0.05$ across all groups). Time dependent receiver operating characteristic curve analysis and decision curve analysis showed that the novel $\mathrm{N}$ stage was superior to the current ypN stage.

Conclusions: The presence of TDs is an independent poor prognostic factor for LARC patients after NCRT. The concomitant presence of TDs and LNM indicates a significantly worse survival, and the addition of TD to LNM may help to better prompt appropriate risk stratification.

Keywords: Tumor deposits (TDs); rectal cancer; neoadjuvant chemoradiotherapy (NCRT); novel stage; prognosis

Submitted Aug 02, 2021. Accepted for publication Nov 17, 2021.

doi: $10.21037 /$ tcr-21-1480

View this article at: https://dx.doi.org/10.21037/tcr-21-1480

^ ORCID: 0000-0001-7522-1949. 


\section{Introduction}

Colorectal cancer (CRC) is the third most common malignancy and the third-leading cause of cancer-related death in both men and women in the United States (1). Neoadjuvant chemoradiotherapy (NCRT) followed by total mesorectal excision is the standard of care for locally advanced rectal cancer (LARC) $(2,3)$. Currently, the American Joint Committee on Cancer (AJCC) TNM staging system (8th edition) is used to assess the prognosis of patients who receive NCRT, with the same categorical definitions as those for patients who do not receive NCRT (4). Tumor deposits (TDs) are defined as isolated tumor lesions in perirectal or mesenteric adipose tissue, far from the leading edge of the tumor and showing no evidence of residual lymph node tissue, but within the lymphatic drainage of the primary carcinoma (3-5). Patients who are TD positive but lymph node metastasis (LNM) negative are classified as N1c according to the 8th edition of the AJCC-TNM staging system (4).

Several previous studies have revealed that preoperative treatment-naive patients with TDs tend to have aggressive clinicopathological features and a worse prognosis (5-8). Additionally, the concomitant presence of TDs and LNM indicated a significantly worse survival, and the prognostic value of TDs alone was similar to that of LNM alone $(5,7,8)$. The number of TDs probably should be considered as LNM when performing $\mathrm{N}$ staging for prompt appropriate risk stratification and may help to better define the duration of adjuvant therapy (9).

NCRT can lead to shrinkage of the primary tumor and metastases, formation of mucous lakes and tissue fibrosis, decreased lymph node retrieval, and a decreased TD count $(10,11)$. In some cases, TDs may result from fragmentation during tumor regression, and islands of tumor cells in the mesentery are remnants of a previously larger tumor that regressed rather than a primary phenomenon $(12,13)$. The prognostic value of TDs may be different from that of treatment-native because of the downstaging effect of NCRT. Some studies have shown that the presence of TDs is associated with a poor treatment response, aggressive clinicopathological features and a poor prognosis in LARC patients after NCRT $(10,12,13)$. A systematic review and meta-analysis including 1,283 patients found that the presence of TDs after NCRT was associated with depth of invasion, lymph node invasion, perineural invasion, synchronous metastasis, and a poor prognosis (10). However, the prognostic value of TDs concomitant with LNM remains controversial for rectal cancer after NCRT for rectal cancer after NCRT. Wang et al. included 550 patients showing that TDs are an independent adverse prognostic factor for LARC after NCRT, particularly for patients with no more than one PLN (14). However, Yu et al. showed that in the current TNM staging system, the classification of TDs in the ypN0 stage was reasonable, while significantly different disease free survival rates were found between the ypN2TD(-) and ypN2TD(+) groups, not for the ypN1group (15). How the prognostic implications of TDs compare with those of LNM remains unclear. This study aimed to evaluate the prognostic value of TDs alone and combined with LNM in LARC patients after NCRT and to reasonably evaluate the addition of TDs to the LNM count.

We present the following article in accordance with the REMARK reporting checklist (available at https://dx.doi. org/10.21037/tcr-21-1480).

\section{Methods}

\section{Data source}

The data were obtained from the Surveillance, Epidemiology and End Results (SEER) 18 (year range, 1975-2016) database and analyzed using SEER*Stat 8.3.8 software. A total of 34,948 patients, according to the 3 rd Edition of International Classification of Diseases for Oncology (ICD-O-3) of the rectum (C20.9) who were treated with NCRT between 2010 and 2016, were identified. After the exclusion of patients with a previous cancer diagnosis $(\mathrm{N}=3,265)$, patients with rectal adenocarcinoma or mucinous or signet-ring cell carcinoma were identified using histology codes (8140 $8144,8210,8211,8260-8263,8440,8480,8481$, and 8490) ( $\mathrm{N}=31,683)$. Excluded patients included those who did not undergo radical surgical resection (Surg Prim Site code $0-28 ; \mathrm{N}=3,856$ ) and those with missing information on TDs $(\mathrm{N}=15,441)$ and lymph nodes $(\mathrm{N}=416)$. After excluding patients for whom the cause-specific death classification was "N/A not first tumor" $(\mathrm{N}=1,120)$, the survival months flag was not incomplete $(\mathrm{N}=140)$, and the $\mathrm{T}$ stage was blank ( $\mathrm{N}=987), 9,620$ patients were included in the study. In this study, cancer-specific survival (CSS) was defined as death caused by rectal cancer. The study was conducted in accordance with the Declaration of 
Helsinki (as revised in 2013).

\section{TD and lymph node information}

With the implementation of the Collaborative Stage Data Collection System Version 02.05 in 2010, TD information was recorded in the SEER database as site-specific factor (SSF) 4, and positive lymph node (PLN) and total lymph node (TLN) information was recorded in the SEER database as the number of positive regional nodes and total regional nodes examined, respectively.

\section{Statistical analysis}

The primary outcome of the study was cancer-specific survival. Patients were divided into 2 groups according to the TD status. Statistical analyses were performed using the table-one package in $\mathrm{R}$ version 3.5 .1 (https://www. r-project.org/) (for Windows) were conducted to identify relationships between TDs and the demographic and clinicopathological characteristics. Categorical variables were grouped based on clinical and pathological findings, and Fisher's exact test was used to compare the results. Survival analyses were performed using the KaplanMeier method and log-rank test. Cox proportional hazards regression analysis was performed for multivariate comparisons, and the final model was generated using backward stepwise selection using the Akaike information criterion. We combined the TD count with LNM and proposed a novel $\mathrm{N}$ stage with five categories according to the Kaplan-Meier method. The novel $\mathrm{N}$ stage and $\mathrm{ypN}$ staging systems were compared with time-dependent AUC and decision curve analysis (DCA) (using the timeROC, ggplot2, and stdca packages). A significance level of 0.05 (two-sided test) was applied throughout.

\section{Results}

\section{Baseline patient characteristics}

Between January 2010 and December 2016, 9,620 LARC patients treated with NCRT who had undergone radical surgery were included and divided into the TD-positive group and the TD-negative groups. The study population comprised of 5,937 men (61.7\%) and 3,683 women (38.3\%), with a median age of 50 years (IQR: $50-67$ years). In this study population, $26(0.3 \%)$ patients were Tis/T0 stage, 1,368 (14.2\%) were T1-2 stage, 8,021 (83.4\%) were T3-4 stage, and 205 (2.1\%) were Tx stage; 6,446 (67.0\%) patients were ypN0 stage, 2,387 (24.8\%) were ypN1 stage, and 787 $(8.2 \%)$ were ypN2 stage; and 9,098 (94.6\%) patients were M0 stage, and 522 (5.4\%) were M1 stage.

The demographic and clinicopathological characteristics of the patients in the TD-positive and TD-negative groups are summarized in Table 1 . In total, 865 patients $(9.0 \%)$ were TD positive, among whom 331 (38.3\%) had ypN1a/b stage tumors, 365 (42.2\%) had ypN1c, and 169 (19.5\%) had ypN2. Compared with the absence of TDs, the presence of TDs was associated with advanced $\mathrm{T}$ stage $(\mathrm{P}<0.001)$,

Table 1 Baseline clinicopathological characteristics in rectal cancer with preoperative radiotherapy

\begin{tabular}{lcc}
\hline Characteristic & TD-negative $(\mathrm{n}=8,755), \mathrm{n}(\%)$ & TD-positive $(\mathrm{n}=865), \mathrm{n}(\%)$ \\
\hline Age, years & $6,022(68.8)$ & \\
$<65$ & $1,733(31.2)$ & $257(29.7)$ \\
$\geq 65$ & & \\
Sex & $5,409(61.8)$ & $528(61.0)$ \\
Male & $3,346(38.2)$ & $337(39.0)$ \\
Female & & \\
Race & $7,072(80.8)$ & $705(81.5)$ \\
White & $722(8.2)$ & $70(8.1)$ \\
Black & $961(11.0)$ & $90(10.4)$ \\
Other & & 0.882 \\
\hline
\end{tabular}

Table 1 (continued) 
Table 1 (continued)

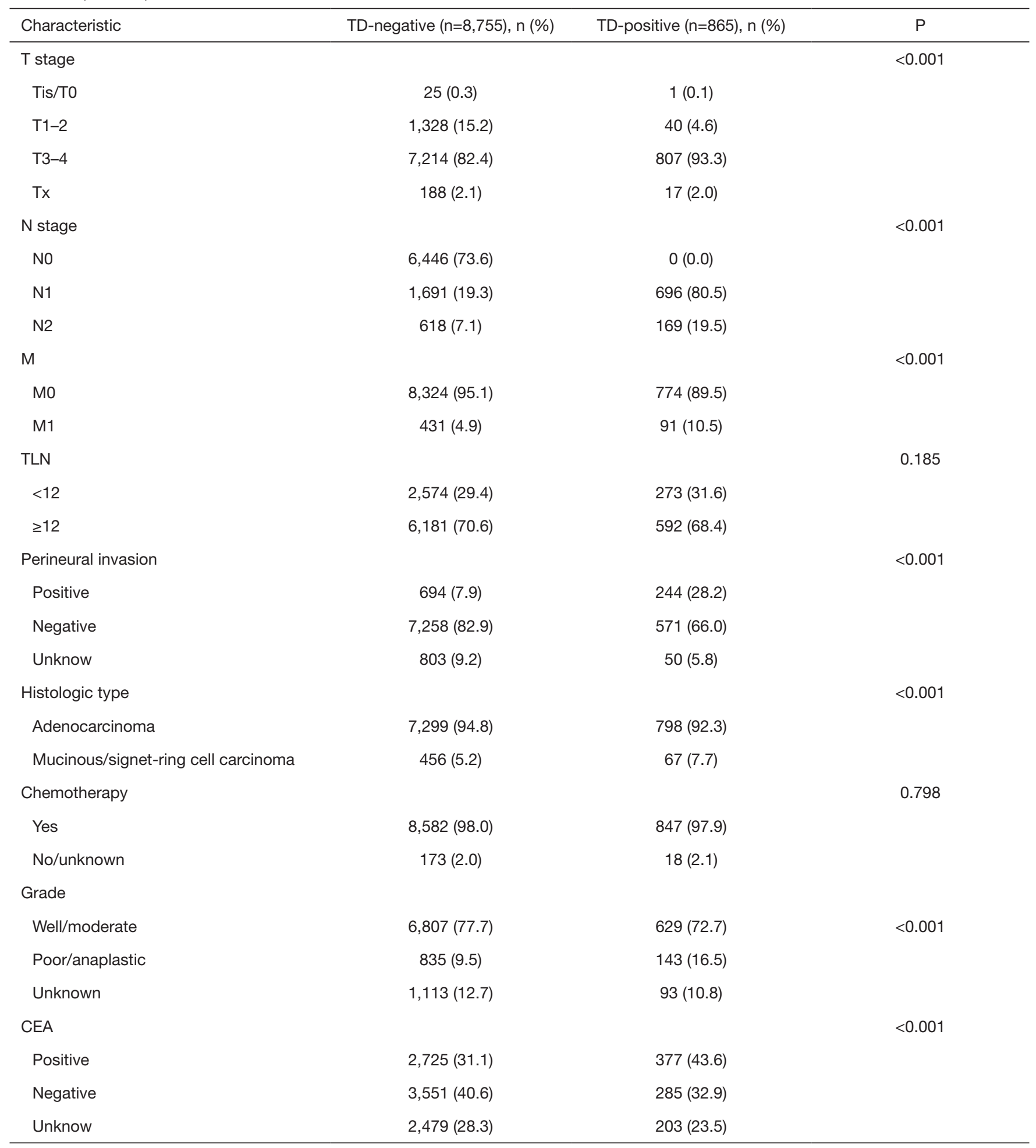

TLN, total lymph node; CEA, carcinoma embryonic antigen. 
advanced $\mathrm{N}$ stage $(\mathrm{P}<0.001)$, a higher tumor grade $(\mathrm{P}<0.001)$, greater perineural invasion $(\mathrm{P}<0.001)$, higher carcinoma embryonic antigen $(\mathrm{CEA})$ positivity $(\mathrm{P}<0.001)$, distant metastases $(\mathrm{P}<0.001)$ and mucinous/signet-ring cell carcinoma $(\mathrm{P}<0.001)$. However, no statistically significant differences were found between the TD-positive and TD-negative groups in age at diagnosis $(\mathrm{P}=0.376)$, race $(\mathrm{P}=0.882)$, sex $(\mathrm{P}=0.687)$, the number of lymph nodes harvested $(\mathrm{P}=0.185)$ or postoperative chemotherapy $(\mathrm{P}=0.798)$.

\section{Prognostic value of TDs in LARC after NCRT}

We investigated the relationship between the clinicopathological characteristics and the CSS of patients with LARC after NCRT using univariate and multivariate analyses. Both the univariate and multivariate analyses indicated that age, $\mathrm{T}$ stage, the LN status, $M$ stage, TDs, total lymph nodes harvested, perineural invasion, the histologic type, chemotherapy, grade, and the CEA status were associated with CSS (Table 2).

To explore the prognostic value of TDs, we analyzed the prognostic implications of TDs in all patients and patient subgroups, including the LNM-, ypN1, ypN2 and M1 subgroups. TDs were a negative prognostic factor for CSS in all patients ( $\mathrm{HR}=2.39,95 \% \mathrm{CI}: 2.04-2.80, \mathrm{P}<0.001$, Figure $1 A$ ), and multivariate analysis showed that TDs were an independent prognostic factor for LARC after NCRT (HR $=1.41,95 \%$ CI: 1.19-1.67, $\mathrm{P}<0.001$, Table 2). Regarding the LN status, TD-positive patients had a higher risk of cancer-specific death than TD-negative patients in the LNM- group ( $\mathrm{HR}=2.43,95 \% \mathrm{CI}: 1.86-3.18, \mathrm{P}<0.001$ ), ypN1 group ( $\mathrm{HR}=2.08,95 \% \mathrm{CI}: 1.61-2.67, \mathrm{P}<0.001)$ and $\mathrm{M} 1$ group ( $\mathrm{HR}=1.51,95 \% \mathrm{CI}: 1.08-2.10, \mathrm{P}<0.001$; Figure $1 B-1 D)$. However, in the ypN2 group, the risk of cancer-specific death was similar in TD-positive and TDnegative patients $(\mathrm{HR}=0.97,95 \% \mathrm{CI}$ : $0.69-1.36, \mathrm{P}=0.84$, Figure $1 E$ ). Patients in the ypN1 and ypN2 groups were further separated into the ypN1a, ypN1b, ypN2a and ypN2b subgroups; in the ypN1a and ypN1b subgroups, TD-positive patients had a worse prognosis than TD-negative patients (Figure S1A,1B), whereas TDpositive and TD-negative patients had similar prognoses in the ypN2a and ypN2b groups (Figure S1C,1D).

\section{Prognostic value of TD and PLN count}

To explore the prognostic value of concurrent TDs and
LNM, patients were divided into the LNM-TD-, LNMTD+, LNM+TD-, and LNM+TD+ groups. In the stratified univariate analysis, patients in the TD-LNM- group had the best CSS (5YCSS, 86.2\%; Figure $1 F$ ), while patients with both TDs and LNM had significantly worse survival than those with only TDs or LNM (5YCSS: 48.2\%, 72.2\%, and $67.8 \%$, respectively; Figure $1 F$ ). These findings suggest that the TD count and PLNs may have similar prognostic value. Therefore, we compared the prognostic value of TD count and PLN count separately. No significant difference was found in survival prognosis between patients with $1 \mathrm{TD}$ and those with 2 or between patients with 3 TDs and those with more. However, the CSS rate of patients with $\geq 3$ TDs was significantly lower than that of patients with 1 or 2 TDs. Therefore, the optimal cutoff value for TD count was 2 (Figure 2A). There were no significant differences in prognosis among patients with a PLN count of 2, 3 or 4-6 (Figure 2B). These findings suggest that the current staging of ypN1b and ypN2a is not reasonable for LARC after NCRT.

\section{Modified pathological N category incorporating TDs}

Based on the Kaplan-Meier survival curves, the 5-year CSS rates were $86.2 \%, 74.7 \%, 62.5 \%, 72.2 \%, 66.4 \%$ and $43.0 \%$ in the ypN0 $(\mathrm{n}=6,446), y p N 1 \mathrm{a}(\mathrm{n}=1,076), y p N 1 b \quad(\mathrm{n}=946)$, ypN1c $(\mathrm{n}=365), y p N 2 a(n=458)$, and ypN2b ( $\mathrm{n}=329)$ groups, respectively (ypN1c vs. ypN1a or ypN1b or ypN2a, ypN1b vs. ypN2a; $>0.05$, Figure $3 A$ ). Because the TD count had a prognostic value similar to that of the PLN count, we combined the TD count with LNM and proposed a novel $\mathrm{N}$ stage with five categories: novel N0 (no regional LNM or TDs), novel N1a (PLN + TD =1), novel N1b (PLN + $\mathrm{TD}=2-4)$, novel N2a (PLN + TD $=5-8)$, and novel N2b $(\mathrm{PLN}+\mathrm{TD} \geq 9)($ Figure 3B,3C). The 5-year CSS rates were $86.2 \%, 77.4 \%, 65.1 \%, 53.8 \%$, and $46.5 \%$ in the novel N0 $(\mathrm{n}=6,446)$, novel N1a $(\mathrm{n}=1,116)$, novel N1b $(\mathrm{n}=1,286)$, novel $\mathrm{N} 2 \mathrm{a}(\mathrm{n}=481)$, and novel N2b ( $\mathrm{n}=291)$ groups, respectively $(\mathrm{P}<0.05$ across all groups; Figure $3 \mathrm{C})$.

\section{Comparison of the CSS predictive accuracy of the novel $N$ stage and current ypN stage}

Time-dependent receiver operating characteristic (timeROC) curve analysis was used to determine which $\mathrm{N}$ stage more accurately predicted CSS. The median timedependent AUC value for the novel $\mathrm{N}$ stage was 0.812 (95\% CI: 0.743-0.908), which was higher than that for the 
Table 2 Univariate and multivariate analyses for the prognostic value of tumor deposits

\begin{tabular}{|c|c|c|c|c|c|c|c|}
\hline Characteristic & \multicolumn{4}{|c|}{ Univariate analysis } & \multicolumn{3}{|c|}{ Multivariate analysis } \\
\hline Age, years & & & & $<0.001$ & & & $<0.001$ \\
\hline$<65$ & $6,630(68.9)$ & 1 & Reference & & 1 & Reference & \\
\hline$\geq 65$ & $2,990(31.1)$ & 1.42 & $1.26-1.60$ & & 1.52 & $1.35-1.71$ & \\
\hline Male & $6,630(68.9)$ & 1 & Reference & & & & \\
\hline Female & $2,990(31.1)$ & 0.93 & $0.83-1.05$ & & & & \\
\hline Race & & & & 0.175 & & & \\
\hline White & $7,777(80.8)$ & 1 & Reference & & & & \\
\hline T stage & & & & $<0.001$ & & & 0.001 \\
\hline Tis/T0 & $26(0.3)$ & 1 & Reference & & 1 & Reference & \\
\hline $\mathrm{T} 1-2$ & $1,368(14.2)$ & 0.79 & $0.25-2.49$ & & 0.59 & $0.19-1.87$ & \\
\hline T3-4 & $8,021(83.4)$ & 1.58 & $0.51-4.91$ & & 0.89 & $0.29-2.78$ & \\
\hline Tx & $205(2.1)$ & 2.18 & $0.61-7.81$ & & 0.90 & $0.25-3.26$ & \\
\hline LN status & & & & $<0.001$ & & & $<0.001$ \\
\hline Negative & $6,811(70.8)$ & 1 & Reference & & 1 & Reference & \\
\hline Positive & 2,809 (29.2) & 2.67 & $2.38-3.00$ & & 2.04 & $1.80-2.31$ & \\
\hline Positive & $865(9.0)$ & 2.39 & $2.04-2.80$ & & 1.41 & $1.19-1.67$ & \\
\hline TLN & & & & 0.009 & & & $<0.001$ \\
\hline$<12$ & 2,847 (29.6) & 1 & Reference & & 1 & Reference & \\
\hline$\geq 12$ & $6,773(70.4)$ & 0.85 & $0.76-0.96$ & & 0.80 & $0.71-0.90$ & \\
\hline Perineural invasion & & & & $<0.001$ & & & $<0.001$ \\
\hline Negative & $7,829(81.4)$ & 1 & Reference & & 1 & Reference & \\
\hline Positive & $938(9.8)$ & 2.86 & $2.47-3.30$ & & 1.82 & $1.56-2.13$ & \\
\hline Unknow & $853(8.9)$ & 1.13 & $0.92-1.38$ & & 1.12 & $0.91-1.37$ & \\
\hline Histologic type & & & & $<0.001$ & & & \\
\hline Adenocarcinoma & 9,097 (94.6) & 1 & Reference & & 1 & Reference & $<0.001$ \\
\hline $\begin{array}{l}\text { Mucinous/signet-ring cell } \\
\text { carcinoma }\end{array}$ & $523(5.5)$ & 1.99 & $1.65-2.40$ & & 1.58 & $1.31-1.91$ & \\
\hline
\end{tabular}

Table 2 (continued) 
Table 2 (continued)

\begin{tabular}{|c|c|c|c|c|c|c|c|}
\hline Characteristic & \multicolumn{4}{|c|}{ Univariate analysis } & \multicolumn{3}{|c|}{ Multivariate analysis } \\
\hline Chemotherapy & & & & & & & 0.003 \\
\hline Yes & $9,429(98.0)$ & 1 & Reference & & 1 & Reference & \\
\hline No/unknown & $191(2.0)$ & 0.59 & $042-0.82$ & & 0.60 & $0.43-0.84$ & \\
\hline Well/moderate & $7,436(77.3)$ & 1 & Reference & & 1 & Reference & \\
\hline Poor/anaplastic & $978(10.2)$ & 2.17 & $1.87-2.51$ & & 1.73 & $1.49-2.01$ & \\
\hline Unknown & $1,206(12.5)$ & 0.86 & $0.70-1.05$ & & 0.84 & $0.69-1.03$ & \\
\hline CEA & & & & $<0.001$ & & & $<0.001$ \\
\hline Unknow & 2,682 (27.9) & 1.17 & $1.01-1.36$ & & 1.09 & $0.94-1.27$ & \\
\hline
\end{tabular}

TLN, total lymph node; CEA, carcinoma embryonic antigen.
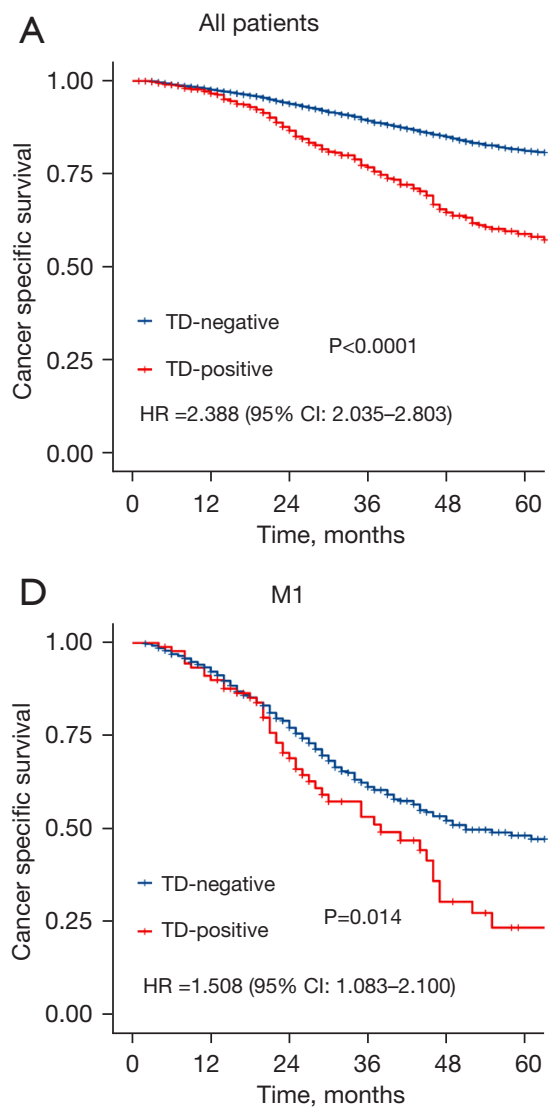

B

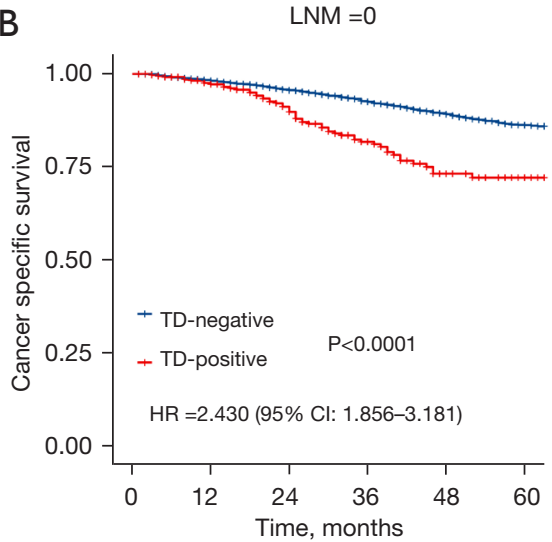

E

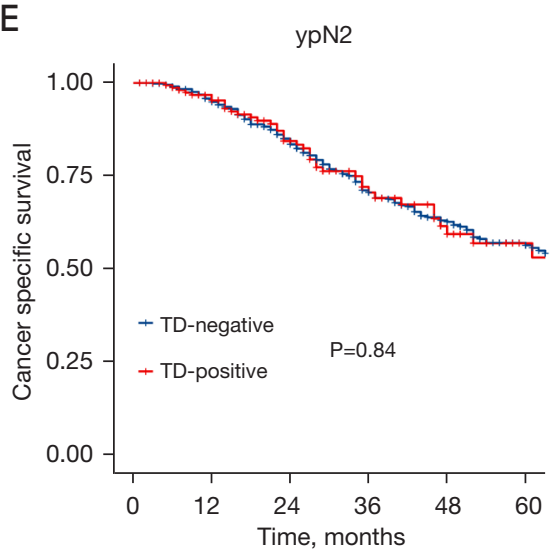

C

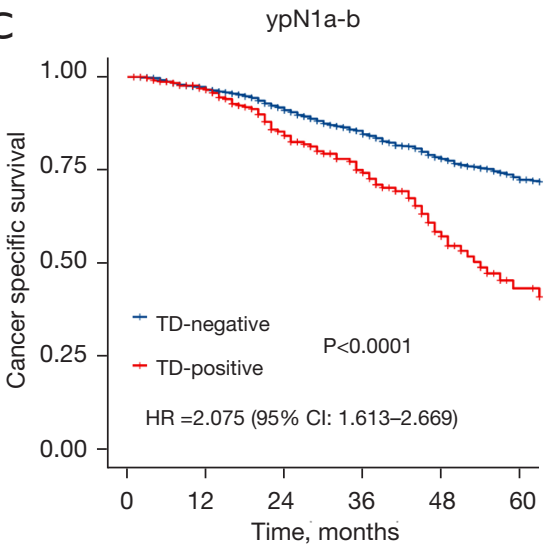

$\mathrm{F}$

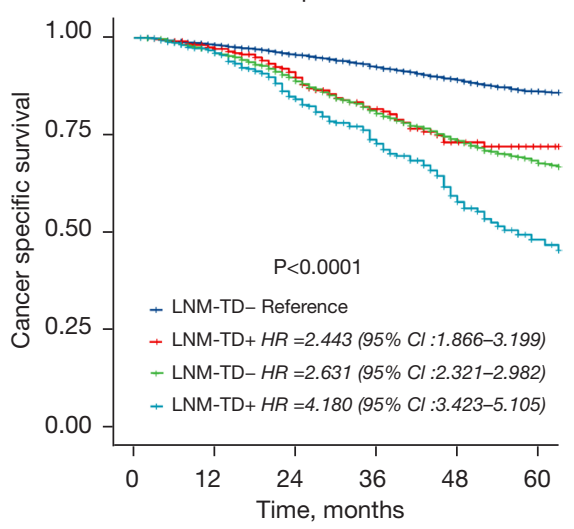

Figure 1 Kaplan-Meier curves of TDs status in overall patients (A), no lymph node metastasis group (B), ypN1a-b group (C), M1 group (D), ypN2 group (E) and Kaplan-Meier curves comparing survival for LNM-TD-, LNM+TD-, LNM-TD+, and LNM+TD+ (F). TD, tumor deposits; LNM, lymph node metastasis. 

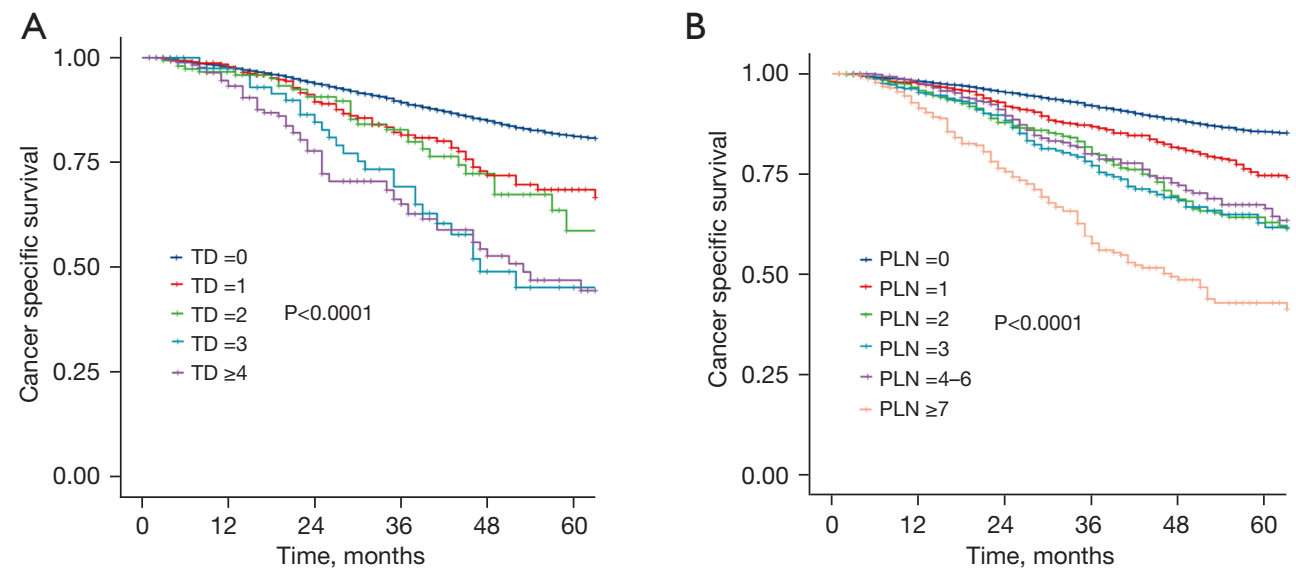

Figure 2 Kaplan-Meier curves of different counts of TDs (A) and positive lymph nodes (B). TD, tumor deposits; PLN, positive lymph node.
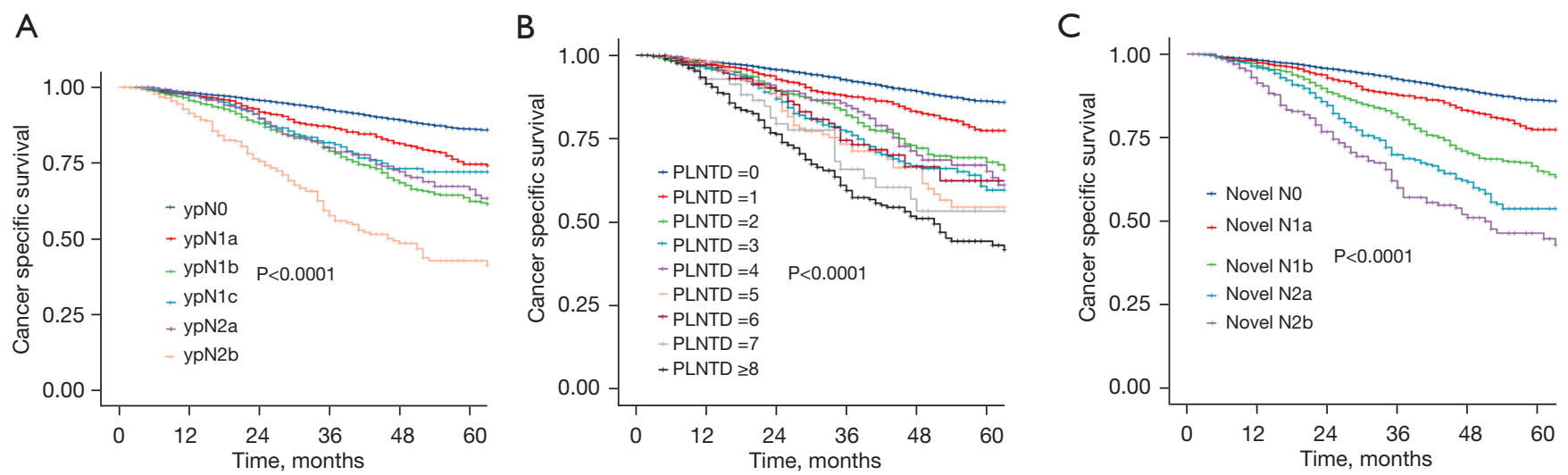

Figure 3 Cancer-specific survival according to the ypN stage (A), combining TD and LNM counts (B) and the novel N stage. PLNTD, the number of positive lymph nodes plus the number of TDs.

current ypN stage (Figure $4 A$ ).

DCA showed the clinical usefulness of each model based on a continuum of potential thresholds for the risk of cancer-related death (x-axis) and net benefit of using the model to stratify patients by risk (y-axis) based on the assumption that no patient would experience cancer-related death. The DCA revealed that the novel $\mathrm{N}$ stage provided superior net benefit compared with the commonly used ypN stage (Figure $4 B$ ).

\section{Discussion}

The results of the present study suggest that TDs are associated with a worse outcome for patients with LARC after NCRT. Additionally, concomitant TDs and LNM were associated with significantly worse CSS than the presence of either TDs or LNM alone. The TD count and PLNs had similar prognostic characteristics, and the TD count was combined with the LNM count to generate a novel $\mathrm{N}$ stage that can accurately predict CSS with better usefulness than the current ypN staging system.

Currently, the TNM staging system is the most frequently used and important prognostic tool to predict the prognosis of CRC (16). The 7th edition of the AJCCTNM staging system includes TDs and proposes $\mathrm{pN} 1 \mathrm{c}$ staging. The current eighth edition of the AJCC staging manual emphasizes the importance of no evidence of residual lymph node, neural, and vascular tissues with other details remaining unchanged (4). Current National Comprehensive Cancer Network (NCCN) guidelines state that the TD count should be recorded in the pathology report and that these tissues should not be counted as 

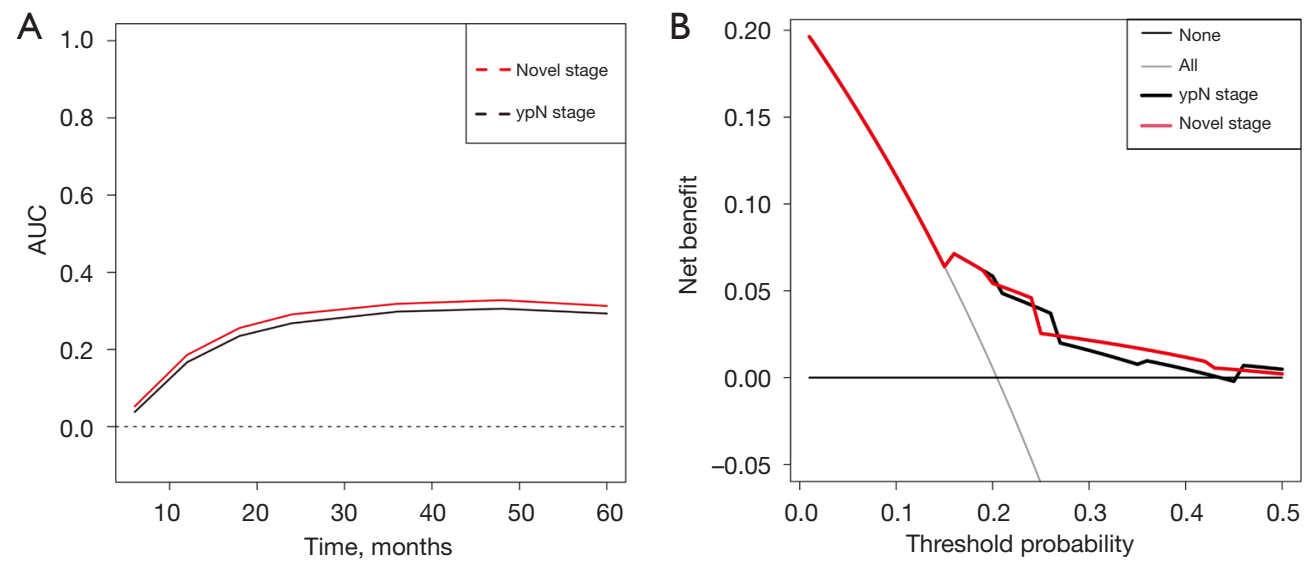

Figure 4 TimeROC and decision curve analysis of the novel $\mathrm{N}$ stage and ypN stage. TimeROC, time-dependent receiver operating characteristic.

metastatic lymph nodes (2).

The origin of TDs, particularly those remaining after NCRT, remains controversial. Some scholars contend that TDs are residual tumors remaining after primary tumor regression (13), while others believe that TDs originate from metastatic lymph nodes or perineural or vascular invasion $(4,5,16)$. Although the origin of TDs is controversial, a consensus exists on the adverse prognosis of TDs for CRC and has been explored in many studies $(5-8,10,13)$. However, it remains unclear how to integrate TDs into the TNM staging system remains unclear, particularly for LARC after NCRT; incorporating the TD count with the LNM count is a possible solution. Mirkin et al. studied 6,624 stage III colon adenocarcinomas and showed that the presence of both TDs and LNM was associated with a significantly worse prognosis. The presence of TDs alone or LNM alone appeared to have a similar prognostic effect. The 5-year survival rates of patients in the TD+LNM+, TD+LNM-, and TD-LNM+ groups were $40.2 \%, 68.1 \%$, and $55.4 \%$, respectively (8). Liu et al. investigated 69,178 cases of CRC in the SEER database and 3,137 cases at Fudan University Shanghai Cancer Center and found that TDs were an independent prognostic factor associated with a shorter overall survival (OS). Furthermore, patients with N1TD+ had a prognosis similar to that of $\mathrm{N} 2$ patients, while $\mathrm{N} 2 \mathrm{TD}+$ patients had a significantly worse prognosis than N2TD- patients. However, the number of TDs did not affect the prognosis. Therefore, the study recommended that TDs should also be considered in the TNM system when LNM is present (7). Delattre et al. reanalyzed the prognostic value of TDs in stage III colon cancer patients in the IDEA France Phase III study and found that the prognostic values of $\mathrm{pN} 1 \mathrm{c}$ and $\mathrm{pN} 1 \mathrm{a} / \mathrm{b}$ were similar and that the presence of TDs was associated with a worse prognosis across all pN stages. That study, in agreement with other studies, suggested that TDs should be integrated into $\mathrm{pN}$ staging to appropriately classify stage III disease. Thus, they proposed a novel TNM staging system combining the TD count with the LNM count, and 35 (2.4\%) patients were restaged as $\mathrm{pN} 2(9)$.

Several studies have assessed the prognostic value of TDs after NCRT in LARC patients, but the conclusions are controversial. In 2011, Song et al. reported the prognostic value of TDs after NCRT in 136 LARC patients. The incidence of TDs was $11.8 \%$, and no significant difference was found in disease-free survival (DFS) or OS between the TD-positive and TD-negative groups $(\mathrm{P}=0.48)$. That study suggested that the ypN1c classification might not apply to LARC patients after NCRT (17). Gopal et al. showed that the presence of TDs was associated with $\mathrm{LNM}(\mathrm{P}=0.035)$, distant metastases $(P=0.006)$ and decreased survival $(\mathrm{P}=0.027)$, and $\mathrm{TD}$-positive patients had worse treatment responses and higher rates of local recurrence $(14,18)$. These findings may explain the associations between TDs and higher recurrence rates and lower survival rates after NCRT. In 2015, Zhang et al. evaluated 310 patients and found that TDs were an independent poor prognostic factor for LARC after NCRT and that TD-positive patients appeared to benefit from adjuvant chemotherapy (19). 
Wei et al. studied 4,813 patients in the SEER database and found that the incidence of TDs was $10.7 \%$; TDs were an independent negative prognostic factor for tumorspecific survival after NCRT in rectal cancer (adjusted HR $=2.25,95 \%$ CI: $1.51-3.35$ ), and survival was significantly lower in the N1c group than in the N0 group (adjusted HR =2.41, 95\% CI: 1.24-4.69) (20). Wang et al. included 550 studies showing that TDs are an independent adverse prognostic factor for LARC after NCRT, particularly for patients with less than one PLN, and TDs should probably be considered one PLN when performing $\mathrm{N}$ staging (14). However, Yu et al. showed that in the current TNM staging system, the classification of TDs in the ypN0 stage was reasonable, while $\mathrm{TD}$ classification at the $\mathrm{ypN}+$ stage was inappropriate (15). A systematic review and meta-analysis including 1,283 patients found that the incidence of TDs ranged from $11.8 \%$ to $44.2 \%$ (mean $23.7 \%$ ), similar to that in untreated patients. The presence of TDs after NCRT was associated with depth of invasion, lymph node invasion, perineural invasion, synchronous metastasis, and a poor prognosis (10).

In the present study, we analyzed data on 9,620 LARC patients after NCRT in the SEER database to evaluate the prognostic value of TDs alone and TDs with LNM and the effect of including both TD presence and count in $\mathrm{pN}$ staging to derive a novel $\mathrm{N}$ stage.

First, patients were divided into TD-positive and TDnegative groups; the presence of TDs was associated with worse clinicopathological features, a finding that was consistent with the results of previous studies $(5,8,10,12,15,19-21)$. Second, multivariate analysis demonstrated that the presence of TDs was an independent poor prognostic factor. Survival analysis showed that the presence of TDs indicated a worse CSS in all patients, as well as in the N1 and M1 groups. The presence of TDs did not have prognostic significance in $\mathrm{N} 2$ patients. Patients with N2 disease after NCRT consistently showed a worse treatment response and advanced disease, and this patient subset had a worse prognosis $(14,19)$, possibly accounting for the lack of a statistically significant interaction between the presence of TDs and CSS in this group.

A systematic review and meta-analysis revealed that omitting TDs in the presence of LNM leads to a loss of valuable prognostic information for untreated patients (5). In our study, no significant interactions with CSS were observed in the LNM+TD- and LNM-TD+ groups, indicating that the TD count may have the same prognostic value as the LNM count. Thus, considering one TD as one LNM and including the total numbers of LNMs and TDs in $\mathrm{N}$ staging is better than placing LNM-negative patients with TDs in the N1c category. Therefore, we propose that the TD count be added to the LNM count to derive a novel $\mathrm{N}$ stage. Both the timeROC and DCA results indicated that the novel $\mathrm{N}$ stage was superior to the current ypN staging, and Kaplan-Meier plots revealed that the prognostic efficacy of the novel $\mathrm{N}$ stage was better than that of the ypN system.

Our findings are supported by the large sample size and the inclusion of cases from 2010 onwards, corresponding with the publication of the seventh edition of the TNM staging system. However, our study has several limitations. First, this retrospective study lacked a rigorous experimental design, which may have caused selection bias, although the inclusion of a sufficient sample size mitigates selection bias. Second, our analysis ignored the size, profile, and distribution of TDs; we focused only on the number of TDs, and these other characteristics must be further explored. Finally, our proposed novel N staging system must be validated in high-quality studies.

\section{Conclusions}

Our findings suggest that the presence of TDs is an independent poor prognostic factor for LARC patients after NCRT. Additionally, the concomitant presence of TDs and LNM indicated a significantly worse survival, and the addition of TD to LNM may help to better prompt appropriate risk stratification.

\section{Acknowledgments}

Funding: This work was supported by the National Natural Science Foundation of China (No. 81972444)

\section{Footnote}

Reporting Checklist: The authors have completed the REMARK reporting checklist. Available at https://dx.doi. org/10.21037/tcr-21-1480

Peer Review File: Available at https://dx.doi.org/10.21037/ tcr-21-1480

Conflicts of Interest: All authors have completed the ICMJE 
uniform disclosure form (available at https://dx.doi. org/10.21037/tcr-21-1480). The authors have no conflicts of interest to declare.

Ethical Statement: The authors are accountable for all aspects of the work in ensuring that questions related to the accuracy or integrity of any part of the work are appropriately investigated and resolved. The study was conducted in accordance with the Declaration of Helsinki (as revised in 2013).

Open Access Statement: This is an Open Access article distributed in accordance with the Creative Commons Attribution-NonCommercial-NoDerivs 4.0 International License (CC BY-NC-ND 4.0), which permits the noncommercial replication and distribution of the article with the strict proviso that no changes or edits are made and the original work is properly cited (including links to both the formal publication through the relevant DOI and the license). See: https://creativecommons.org/licenses/by-nc-nd/4.0/.

\section{References}

1. Siegel RL, Miller KD, Goding Sauer A, et al. Colorectal cancer statistics, 2020. CA Cancer J Clin 2020;70:145-64.

2. Benson AB, Venook AP, Al-Hawary MM, et al. NCCN Guidelines Insights: Rectal Cancer, Version 6.2020. J Natl Compr Canc Netw 2020;18:806-15.

3. Glynne-Jones R, Wyrwicz L, Tiret E, et al. Rectal cancer: ESMO Clinical Practice Guidelines for diagnosis, treatment and follow-up. Ann Oncol 2017;28:iv22-40.

4. Nicholls RJ, Zinicola R, Haboubi N. Extramural spread of rectal cancer and the AJCC Cancer Staging Manual 8th edition, 2017. Ann Oncol 2019;30:1394-5.

5. Nagtegaal ID, Knijn N, Hugen N, et al. Tumor Deposits in Colorectal Cancer: Improving the Value of Modern Staging-A Systematic Review and Meta-Analysis. J Clin Oncol 2017;35:1119-27.

6. Lino-Silva LS, Xinaxtle DL, Salcedo-Hernández RA. Tumor deposits in colorectal cancer: the need for a new "pN" category. Ann Transl Med 2020;8:733.

7. Liu F, Zhao J, Li C, et al. The unique prognostic characteristics of tumor deposits in colorectal cancer patients. Ann Transl Med 2019;7:769.

8. Mirkin KA, Kulaylat AS, Hollenbeak CS, et al. Prognostic Significance of Tumor Deposits in Stage III Colon Cancer. Ann Surg Oncol 2018;25:3179-84.
9. Delattre JF, Cohen R, Henriques J, et al. Prognostic Value of Tumor Deposits for Disease-Free Survival in Patients With Stage III Colon Cancer: A Post Hoc Analysis of the IDEA France Phase III Trial (PRODIGE-GERCOR). J Clin Oncol 2020;38:1702-10.

10. Lord AC, Graham Martínez C, D'Souza N, et al. The significance of tumour deposits in rectal cancer after neoadjuvant therapy: a systematic review and metaanalysis. Eur J Cancer 2019;122:1-8.

11. Trakarnsanga A, Gönen M, Shia J, et al. Comparison of tumor regression grade systems for locally advanced rectal cancer after multimodality treatment. J Natl Cancer Inst 2014;106:dju248.

12. Fernández-Aceñero MJ, Estrada Muñoz L, Sastre Varela J, et al. Prognostic influence of histopathological regression patterns in rectal adenocarcinoma receiving neoadjuvant therapy. J Gastrointest Oncol 2017;8:49-54.

13. Perez RO, Habr-Gama A, Smith FM, et al. Fragmented pattern of tumor regression and lateral intramural spread may influence margin appropriateness after TEM for rectal cancer following neoadjuvant CRT. J Surg Oncol 2014;109:853-8.

14. Wang Y, Zhang J, Zhou M, et al. Poor prognostic and staging value of tumor deposit in locally advanced rectal cancer with neoadjuvant chemoradiotherapy. Cancer Med 2019;8:1508-20.

15. Yu L, Xu T, Zhang L, et al. Tumor Deposits Should Not Be Ignored in the AJCC TNM Staging System for ypN(+) Stage Rectal Cancer with Neoadjuvant Chemoradiotherapy. J Gastrointest Surg 2020;24:2298-301.

16. Frankel WL, Jin M. Serosal surfaces, mucin pools, and deposits, oh my: challenges in staging colorectal carcinoma. Mod Pathol 2015;28 Suppl 1:S95-108.

17. Song JS, Chang HJ, Kim DY, et al. Is the N1c category of the new American Joint Committee on cancer staging system applicable to patients with rectal cancer who receive preoperative chemoradiotherapy? Cancer 2011;117:3917-24.

18. Gopal P, Lu P, Ayers GD, et al. Tumor deposits in rectal adenocarcinoma after neoadjuvant chemoradiation are associated with poor prognosis. Mod Pathol 2014;27:1281-7.

19. Zhang LN, Xiao WW, Xi SY, et al. Tumor deposits: markers of poor prognosis in patients with locally advanced rectal cancer following neoadjuvant chemoradiotherapy. Oncotarget 2016;7:6335-44.

20. Wei XL, Qiu MZ, Zhou YX, et al. The clinicopathologic 
relevance and prognostic value of tumor deposits and the applicability of N1c category in rectal cancer with preoperative radiotherapy. Oncotarget 2016;7:75094-103.

21. Wang S, Guan X, Ma M, et al. Reconsidering the

Cite this article as: $\mathrm{Xu} \mathrm{T,} \mathrm{Yu} \mathrm{Z,} \mathrm{Zhang} \mathrm{Q,} \mathrm{Liu} \mathrm{B,} \mathrm{Li} \mathrm{Y,} \mathrm{Wang} \mathrm{F.}$ Prognostic and staging value of tumor deposits in patients with rectal cancer after neoadjuvant chemoradiotherapy. Transl Cancer Res 2021;10(12):5028-5039. doi: 10.21037/tcr-21-1480 prognostic significance of tumour deposit count in the TNM staging system for colorectal cancer. Sci Rep 2020;10:89. 
A

ypN1a

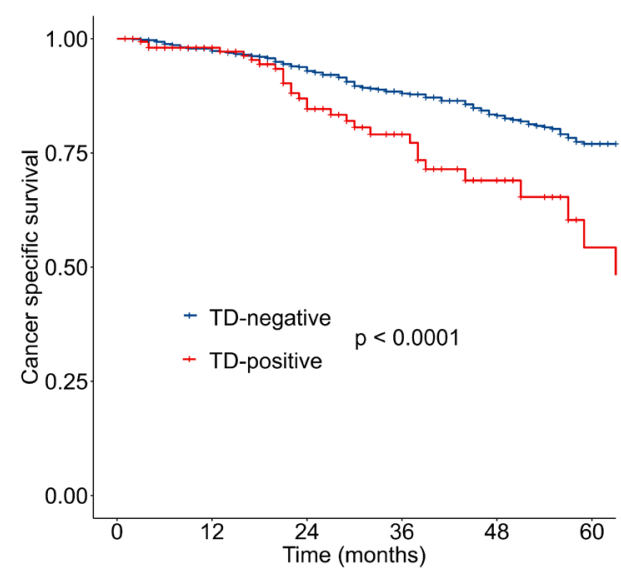

C

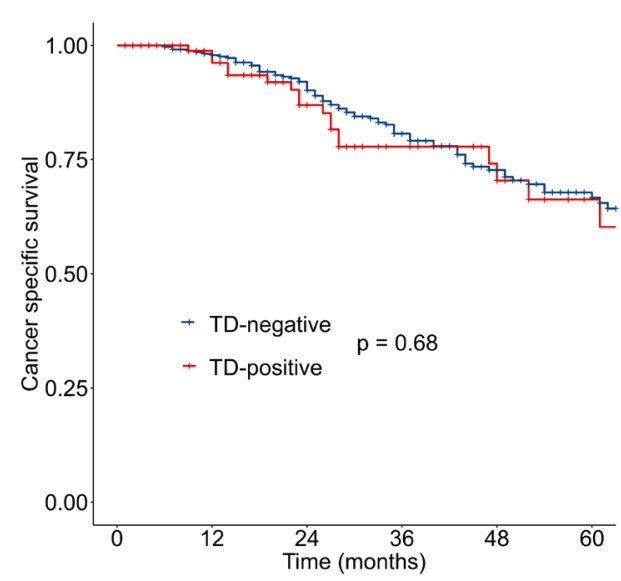

B $\quad$ ypN1b

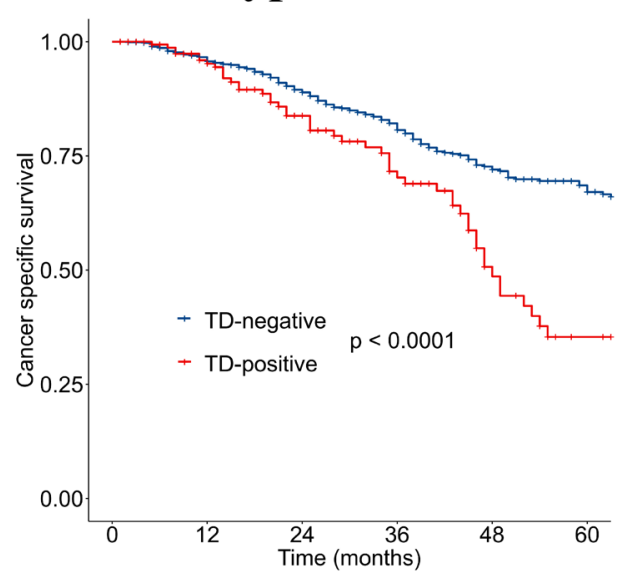

D

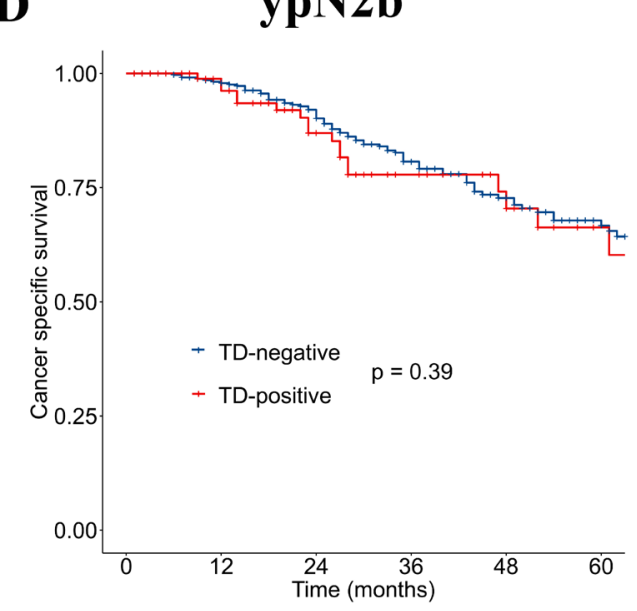

Figure S1 Kaplan-Meier curves of TDs status in ypN1 subgroup (A,B) and ypN2 subgroup (C,D). TD, tumor deposits. 